\title{
Constraints for Service Contracts ${ }^{\star}$
}

\author{
Maria Grazia Buscemi ${ }^{1}$, Mario Coppo ${ }^{2}$, \\ Mariangiola Dezani-Ciancaglini ${ }^{2}$, and Ugo Montanari ${ }^{3}$ \\ 1 IMT Institute for Advanced Studies, Lucca, Italy \\ 2 Dipartimento di Informatica, Università di Torino, Italy \\ 3 Dipartimento di Informatica, Università di Pisa, Italy
}

\begin{abstract}
This paper focuses on client-service interactions distinguishing between three phases: negotiate, commit and execute. The participants negotiate their behaviours, and if an agreement is reached they commit and start an execution which is guaranteed to respect the interaction scheme agreed upon. These ideas are materialised through a calculus of contracts enriched with semiringbased constraints, which allow clients to choose services and to interact with them in a safe way. A concrete representation of these constraints with logic programs is straightforward, thus reducing constraint solution (and consequently the establishment of a contract) to the execution of a logic program.
\end{abstract}

\section{Introduction}

Communicating systems have attracted a lot of interest since the eighties in several areas of computer science. In particular, verification methods have been studied in depth, given the difficulty of correctly designing communication protocols. Very successful has been the decision to abstract out from the actual data and their algorithmic complexity, and to focus on communication properties. Typically, the number of states of a system becomes finite (while possibly very large) and verification techniques based on model checking static analysis become feasible, and quite effective. The need of programming and maintaining eternal, highly decentralised systems (e.g. those considered by the FP7-FET initiative Forever Yours) has emphasised the need to extend to run time as much as possible the verification activity. The expected scenario shows the interacting partners to undergo a verification step: if it succeeds the interaction will be correct. The typical property to check is lack of deadlock, more precisely, stuck-freedom [20]: there are no messages waiting forever to be sent or sent messages which are never received, thus assuring that the interaction between partners will successfully end.

The key idea is to associate to a process an abstract description of its behaviour, and to check if the pairs ${ }^{4}$ of processes which are expected to communicate do match. The extended version of [9] discusses several different approaches to the global and local descriptions of communication protocols. The most common styles used to model abstract process behaviour are session types and behavioural contracts.

Sessions and session types (first introduced in [22]) are built on pi-calculus [18]: the key idea is that types include information about the sequences of messages sent and received, and that type correctness, as defined by suitable type checking rules, assures

\footnotetext{
* Work partially funded by the MIUR Project IPODS and by the EU IST Project ASCENS.

${ }^{4}$ The most common arrangement consists of pair of processes, but multi-party communication is also considered.
} 
stuck-freedom. We refer to [12] and [23] for overviews.

Behavioural contracts are CCS-like processes which describe the communications between clients and services. Many recent papers focus on the compatibility between clients and services and the safe replacements of services. The necessary control on communications is achieved by explicit interfaces [15] and message filtering [10]. Also suitable centralised or decentralised coordination strategies like orchestration [19] or choreography [6] are employed to guarantee safe communication.

There is a large literature on conditions for assuring stuck freedom in process calculi, see [14] and the references there. In particular [13, 1] deal with the case of sessions.

We criticise the existing approaches on two grounds: (i) the result of the static analysis is on-off: either the interaction is acceptable or not. In most cases, an acceptable communication could be achieved, even if less appealing, by restricting partner behaviours. Ideally, the behaviour should be restricted the least, compatibly with stuckfreedom. Also, the execution should be monitored in such a way that only the harmless alternatives are left open; (ii) the verification process must be repeated for every pair of partners. It would be more convenient to split it into two parts: a compilation step, to be executed at deployment time, where the abstract behaviour is determined, and a matching step to be executed at run time, for every pair of partners willing to communicate.

We believe that an approach based on constraints would be able to overcome these difficulties: (i) the compilation step should generate a constraint modelling the behaviour; (ii) the matching step should be simply constraint composition, successful only if the resulting constraint is satisfiable; (iii) the actual execution should be monitored by ask-like guards, reminiscent of concurrent constraint programming [21]. The advantage of a constraint based approach is clear: the necessary constraints can be built inductively at compile time, composed at matching time and tested at run time taking advantage of concepts well-studied in the area of constraint programming.

To the best of our knowledge, the idea of using constraints for negotiating which interactions to choose, hence avoiding deadlocks and assuring success, is novel. Note that the kind of constraints we need is by no means peculiar: a constraint is essentially a set of computed answer substitutions of logic programming, once the Herbrand signature is suitably chosen. Similar constraints are used in [11] to assure quality of services.

We present our constraint system as an instantiation of the class of named constraint semirings [7], which have been originally proposed by two of the authors as the underlying structure of the cc-pi calculus [7], a process calculus for modelling agreements on non-functional parameters in a service oriented scenario. The target calculus we propose is close in spirit to the cc-pi calculus, except for the fact that the primitives of our target calculus are meant to model two-party interactions. [8] presents a prioritised version of cc-pi calculus, in which the non-deterministic choice is replaced by an operation that allows selecting an action if the corresponding constraint has a priority over the constraints of the alternative branches. Though priorities are assigned following different criteria, our optimised semantics is inspired by the prioritised cc-pi calculus.

Constraint semirings [3] are semirings with an idempotent additive operation and a commutative multiplicative operation. They generalise boolean algebra and are equipped with a partial ordering with 1 as maximal and 0 as minimal element. Semiring values model constraints: larger values are less constraining, multiplication means combina- 
tion of constraints, sum returns the least upper bound. Constraint semirings are meant to define soft constraints, namely constraints which do not return only true or false, but more informative values instead (e.g., degree of preference, cost), thus allowing to extend paradigms such as concurrent constraint programming and constraint logic programming. In [5] a version of the soft concurrent constraint programming has been proposed for specification of SLA negotiations, basically with the same goal of the cc-pi calculus. Named constraint semirings in addition come equipped with an algebra of name permutations, which allows characterising the set of relevant names of a constraint as the support of a permutation, that is basically the minimum set of names that are changed by the permutation. Named constraint semirings inherit from ordinary constraints on the boolean semiring properties and efficient algorithms, like constraint propagation and dynamic programming.

As mentioned above, here we take advantage of a quite simple and standard named constraint semiring: the one employed by logic programming, where the Herbrand signature contains as many unary operations as actions and a constant to model termination. In the context of this paper we will use the term 'variable' rather than 'name' in conformance with the standard notation of logic programming. The semiring values are sets of assignments with Herbrand terms to all the variables. However, only the assignments to the tuples of variables forming the support are relevant. Existential quantification adds all the assignments where the restricted variable is assigned in all possible ways. The correspondence with logic programming is quite simple: given a set of clauses and a goal $P\left(x_{1}, \ldots, x_{n}\right)$, its semantics in terms of our constraint system is the set of all the ground instantiations of the goal which satisfy the clauses. The support is the set $\left\{x_{1}, \ldots, x_{n}\right\}$ or smaller. Goal composition is multiplication, while multiple clauses for the same goal model sum. Variables appearing in the body but not in the head of a clause are existentially quantified. In our notation, the effect of recursive clauses is obtained by an explicit fixpoint operator.

Our approach distinguishes three phases: NCE, Negotiate, Commit, Execute, where agents negotiate certain desired behaviours, but without any guarantee of success. However, if and when an agreement is reached (commit), under certain conditions a coordinated computation of the involved agents can start, which is guaranteed to have the properties agreed upon in the negotiation phase. We present a simple source calculus with client and service processes and with a semantics given in terms of labelled transition systems: the clients are recursive and can place nested service calls, while services are permanent (namely a service is not consumed by a call) and nonrecursive. The calculus is nondeterministic, with external choices (à la Milner [17] or, similarly, in the style of nondeterministic choices in logic programming), and a client-service behaviour which allows for more choices is considered better, provided they are stuck-free. A target calculus is then defined, where clients and services are compiled to, augmenting them with named constraint semirings, which encode their behaviour.

To understand our approach, let us first consider the case of a client without nested calls and with a single service. The source client is then compiled into a target client combined with a constraint with just one variable in its support, say $x$. The constraint is thus just a set of traces, representing the behaviour of the client. The compiled code is very similar to the source, except that its choices are guarded by check constructs, 
similar to the ask constructs of concurrent constraint programming, which enable the corresponding continuations only if the global constraint allows it. The source service is also compiled, yielding a constraint on $y$ which represents its behaviour, but no check guards are included. The negotiation phase consists of multiplying the two constraints, and their result with the constraint ${ }^{5} x=y$. The resulting constraint contains exactly all the executions of the source client-service system which are not stuck. If the constraint is not 0 , i.e. if it is not the empty set, the commit takes place, and the execution phase can start. Thanks to the check guards, the traces possible for the target client-service system are exactly those satisfying the constraint. Notice that while the client (service) compilation is static, and thus it does not fit in the NCE scheme, it does not depend on the particular service (client) partner it will be matched to. Thus the open endless requirement is here satisfied by the possibility of deploying new services (clients) without the need of any further modification of the existing services (clients).

For instance, let $T$ be a client which offers the choice between co-actions $\bar{\alpha}$ and $\bar{\beta}$, and $S$ be a service which offers the choice between actions $\alpha$ and $\gamma$, and only co-actions and actions interact successfully. Therefore $T$ and $S$ can safely interact only choosing $\bar{\alpha}$ and $\alpha$, respectively. In our calculus we represent them as $T=\bar{\square} \cdot \bar{\alpha} \cdot \bar{\otimes} \cdot \mathbf{0}+\bar{\beta} \cdot \bar{\nabla} \cdot \mathbf{0}$ and $S=\square . \alpha \cdot \otimes+\gamma \cdot \otimes$, where $\bar{\square}$ is service call, $\bar{\nabla}$ is call end, and $\square, \otimes$ are service and end acceptance. The interactions offered by $T$ and $S$ are represented, respectively, by the constraints $c=(x=\alpha($ end $)) \oplus(x=\beta($ end $))$ and $d=(y=\alpha($ end $)) \oplus(y=\gamma($ end $))$ (noting that we write constraints using only actions). It holds that $c \otimes d \otimes(x=y)=$ $(x=\alpha($ end $)) \otimes(y=\alpha($ end $)) \otimes(x=y) \neq 0$, which reflects the fact that the only successful interaction between $T$ and $S$ is (over) $\alpha$.

Let us now consider the general case of a client with nested calls and several services. Services are compiled in the usual way. The behaviour of the client, instead, must be represented by a constraint with several variables in the support. In fact, different service calls may not be independent: imagine that the client makes a choice in an inner call which must be matched by the corresponding service. Then the client returns to the outer level and makes another choice which must be matched this time by the service corresponding to the outer call. The two choices may be dependent, and this requirement is represented by a constraint with a two-variable support. Thus the ability of the constraint system of representing sets of tuples of traces allows us to guarantee stuckfreedom for complex client-service pairs, which at the best of our knowledge have not been considered in the literature by now.

An example of this kind of clients is $\bar{\square} \cdot \bar{\alpha} \cdot \bar{\square} \cdot \bar{\beta} \cdot(\bar{\gamma} \cdot \bar{\nabla} \cdot \bar{\delta} \cdot \bar{\nabla} \cdot \mathbf{0}+\bar{\mu} \cdot \bar{\otimes} \cdot \bar{\rho} \cdot \bar{\nabla} \cdot \mathbf{0})$, where the choices made by the two nested calls depend on each other. Such a dependency can arise, for instance, when modelling a traveller who asks both for a flight $(\bar{\alpha})$ to an airline company and for a room $(\bar{\beta})$ to a hotel in two alternative different dates. The client request (room, flight) are represented respectively by actions $\bar{\gamma}, \bar{\delta}$ for one date and $\bar{\mu}, \bar{\rho}$ for the other date. This client offers then the choice between the pairs of traces $<\alpha(\delta($ end $)), \beta(\gamma($ end $))>$ and $<\alpha(\rho($ end $)), \beta(\mu($ end $))>$. The resulting constraint

$$
\left(x_{1}=\alpha(\delta(\text { end }))\right) \otimes\left(x_{2}=\beta(\gamma(\text { end }))\right) \oplus\left(x_{1}=\alpha(\rho(\text { end }))\right) \otimes\left(x_{2}=\beta(\mu(\text { end }))\right),
$$
with support $\left\{x_{1}, x_{2}\right\}$, obliges the run of related service call to be coherent.

\footnotetext{
${ }^{5}$ Constraint $x=y$ has support $\{x, y\}$ and contains all assignments with the same term for $x$ and $y$. In logic programming it is specified by the goal $e q(x, y)$ with the clause eq $(\mathrm{x}, \mathrm{x})$ :-.
} 
Paper summary. In the paper, the source and the target calculus are the content of $\S 2$ and $\S 3$, respectively. $\S 4$ presents the compilation from the first to the second calculus and states its soundness and completeness. $\$ 5$ shows how to use the constraints in order to get most liberal interactions. Lastly $§ 6$ discuss some future developments.

\section{Source Calculus}

In choosing the source calculus we were guided by the requirement of having a minimal number of process operators to represent one client with (nested) service calls and a set of available services offering finite interactions. For this reason communications are atomic actions, all choices are external, client processes are recursive and can do nested calls, services are permanent, non recursive and each instance of a service can accept exactly one call. Let $\mathcal{A}$ be an infinite set of actions (ranged over by $\alpha, \beta, \ldots$ ) and Source client processes

$$
\begin{array}{l|l|l|l|l|l|l}
P:=\bar{\square} . P & \bar{\alpha} . P & P+P & \operatorname{rec} p . P & \bar{\nabla} . P & p & \mathbf{0}
\end{array}
$$

Source service processes

$$
Q:=\alpha \cdot Q|Q+Q| \otimes
$$

Table 1. Syntax of source client and service processes.

$\overline{\mathcal{A}}$ be an infinite set of disjoint co-actions (ranged over by $\bar{\alpha}, \bar{\beta}, \ldots$ ), with as usual $\overline{\bar{\alpha}}=\alpha$. The syntax of client and service processes is the content of Table 1. For the sake of readability we assume that client processes offer co-actions and service processes offer actions, + is the external choice, $\bar{\square}$ is service call and $\bar{\nabla}, \otimes$ are end of call for client and service processes, respectively.

Recursive processes cannot have nested calls (namely, service calls cannot occur under the scope of the rec operator). We will consider recursive processes modulo fold/unfold, i.e. we identify the processes rec p.P and $P[$ rec p.P/p $]$.

A client is a client process of the shape $\bar{\square}$.P. A client is balanced if each $\bar{\square}$ is followed by exactly one corresponding $\bar{\nabla}$ in each branch. We use $T$ to range over balanced clients.

A service is a service process prefixed by $\square$ (representing call acceptance), i.e. it has the shape $\square . Q$. We use $S$ to range over services.

We consider systems formed by one balanced client and a set $\mathbb{S}_{s}$ of available services. The interaction between a client and a service is represented by boxing, i.e. enclosing in square brackets, the parallel composition of the client process which follows

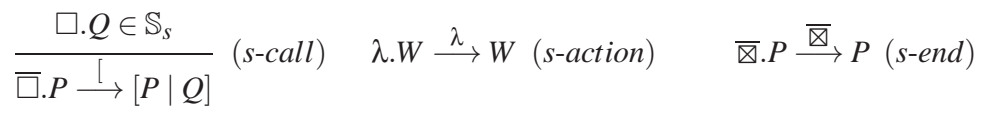

$$
\begin{aligned}
& \frac{W \stackrel{\phi}{\longrightarrow} W^{\prime}}{W+W_{1} \stackrel{\phi}{\longrightarrow} W^{\prime}} \text { (s-choice) } \quad \frac{P \stackrel{\bar{\alpha}}{\longrightarrow} P^{\prime} \quad Q \stackrel{\alpha}{\longrightarrow} Q^{\prime}}{P\left|Q \stackrel{\alpha}{\longrightarrow} P^{\prime}\right| Q^{\prime}} \text { (s-interaction) } \\
& \frac{P \stackrel{\bar{\otimes}}{\longrightarrow} P^{\prime} \quad Q \downarrow_{\otimes}}{[P \mid Q] \stackrel{]}{\longrightarrow} P^{\prime}}(s-u p) \frac{U \stackrel{\psi}{\longrightarrow} U^{\prime}}{[U] \stackrel{\psi}{\longrightarrow}\left[U^{\prime}\right]}(\text { s-box }) \quad \frac{V \stackrel{\psi}{\longrightarrow} V^{\prime}}{V\left|Q \stackrel{\psi}{\longrightarrow} V^{\prime}\right| Q} \text { (s-parallel) }
\end{aligned}
$$

Table 2. LTS for the source calculus 
the call with the interacting service process (see rule (s-call) in Table 2). A client is always interacting with the "copy" of a service in the innermost "box" and outermost instances of services have to wait until an $(s-u p)$ rule is applied. Therefore by reducing a balanced client we will get a box containing either the parallel composition of a client process and a service process or the parallel composition of a box and a service process. We get then the following syntax for systems $V$ :

$$
V::=T|[U]| \mathbf{0} \quad U::=P|Q|[U] \mid Q
$$

Since we want to compare reductions in the source and in the target calculus we give the operational semantics of both calculi via labelled transition systems (LTSs). We say that the process $Q$ exhibits the service end (notation $Q \downarrow \nabla$ ) if either $Q$ is $\otimes$ or $Q$ is the sum of two processes one of which exhibits the service end.

Table 2 gives the reduction rules for the source calculus, where $W$ denotes a client process or a service process, $\lambda \in \mathcal{A} \cup \overline{\mathcal{A}}, \phi \in\{[, \bar{\nabla}\} \cup \mathcal{A} \cup \overline{\mathcal{A}}$ and $\psi \in\{[],\} \cup \mathcal{A}$. The symmetric rule with respect to + has been omitted. Rule (s-interaction) is non standard since $\alpha$ labels the transition in the conclusion, but this does not harm the intended semantics of the calculus. In fact processes do not compose in parallel, systems do not emit outputs, hence no further synchronisation on $\alpha$ is possible. Rule $(s-u p)$ terminates the execution of a call leaving the client process which follows this call one nesting level up.

Let $\sigma$ be a strings on $\{[],\} \cup \mathcal{A}$. We define $V \stackrel{\sigma}{\Longrightarrow} V^{\prime}$ if

- either $\sigma=\varepsilon$ and $V=V^{\prime}$

- or $\sigma=\psi \sigma^{\prime}$ and $V \stackrel{\psi}{\longrightarrow} \stackrel{\sigma^{\prime}}{\Longrightarrow} V^{\prime}$.

We write $V \Downarrow^{\text {may }}$ if $\exists \sigma$ such that $V \stackrel{\sigma}{\Longrightarrow} \mathbf{0}$, i.e. if the system $V$ can reduce to the inert client $\mathbf{0}$.

Example 1. A possible reduction of the client $T=\bar{\square} \cdot \bar{\alpha} \cdot \bar{\square} \cdot \bar{\beta} \cdot(\bar{\gamma} \cdot \bar{\nabla} \cdot \bar{\delta} \cdot \bar{\nabla} \cdot \mathbf{0}+\bar{\mu} \cdot \bar{\nabla} \cdot \bar{\rho} \cdot \bar{\otimes} \cdot \mathbf{0})$ with a set of services including $\{\square . \alpha . \delta . \otimes, \square . \beta .(\gamma \cdot \otimes+v . \otimes)\}$ is:

$$
\begin{aligned}
& T \stackrel{[}{\longrightarrow}[\bar{\alpha} \cdot \bar{\square} \cdot \bar{\beta} \cdot(\bar{\gamma} \cdot \bar{\otimes} \cdot \bar{\delta} \cdot \bar{\nabla} \cdot \mathbf{0}+\bar{\mu} \cdot \bar{\nabla} \cdot \bar{\rho} \cdot \bar{\otimes} \cdot \mathbf{0}) \mid \alpha \cdot \delta \cdot \otimes] \\
& \stackrel{\alpha}{\longrightarrow}[\bar{\square} \cdot \bar{\beta} \cdot(\bar{\gamma} \cdot \bar{\nabla} \cdot \bar{\delta} \cdot \bar{\nabla} \cdot \mathbf{0}+\bar{\mu} \cdot \bar{\nabla} \cdot \bar{\rho} \cdot \bar{\nabla} \cdot \mathbf{0}) \mid \delta \cdot \otimes] \\
& \stackrel{[}{\longrightarrow}[[\bar{\beta} \cdot(\bar{\gamma} \cdot \bar{\otimes} \cdot \bar{\delta} \cdot \bar{\otimes} \cdot \mathbf{0}+\bar{\mu} \cdot \bar{\otimes} \cdot \bar{\rho} \cdot \bar{\nabla} \cdot \mathbf{0}) \mid \beta \cdot(\gamma \cdot \otimes+v \cdot \otimes)] \mid \delta \cdot \otimes] \\
& \stackrel{\beta}{\longrightarrow}[[(\bar{\gamma} \cdot \bar{\nabla} \cdot \bar{\delta} \cdot \bar{\otimes} \cdot \mathbf{0}+\bar{\mu} \cdot \bar{\nabla} \cdot \bar{\rho} \cdot \bar{\otimes} \cdot \mathbf{0}) \mid(\gamma \cdot \otimes+v \cdot \otimes)] \mid \delta \cdot \otimes] \\
& \stackrel{\gamma}{\longrightarrow}[[\bar{\nabla} . \bar{\delta} . \bar{\nabla} . \mathbf{0} \mid \otimes] \mid \delta . \otimes] \stackrel{]}{\longrightarrow}[\bar{\delta} . \bar{\nabla} . \mathbf{0} \mid \delta . \otimes] \stackrel{\delta}{\longrightarrow}[\bar{\nabla} . \mathbf{0} \mid \otimes] \stackrel{]}{\longrightarrow} \mathbf{0}
\end{aligned}
$$

\section{Target Calculus}

The target calculus enriches the source calculus by introducing constraints, which are meant to prevent clients and services from initiating interactions that will eventually lead to deadlocks. The constraints we adopt coincide with those used in logic programming and form a named constraint semiring [7], which is a constraint semiring [3] with a notion of relevant names (here represented by variables) that allows plugging constraints into languages with an explicit concept of names. Below we recall some concepts that are used in the paper. We refer to $[7,3,2]$ for a complete treatment. 


\subsection{Constraints}

Assume a set of variables $\mathcal{V}$, ranged over by $x, y, \ldots$ Let $\Sigma$ be a signature consisting of monadic functions representing actions plus a constant end (expressing successful end):

$$
\Sigma=\{\alpha(-), \text { end } \mid \alpha \in \mathcal{A}\}
$$

A term is any expression that can be obtained from $\mathcal{V}$ and $\Sigma$; a ground term is a term that does not contain variables. The Herbrand Universe $\mathcal{H}$, ranged over by $h$, is the set of ground terms over $\Sigma$. A ground assignment is a total function $s$ that maps variables to ground terms, namely $s: \mathcal{V} \rightarrow \mathcal{H}$. We define $s[h / x]$ by $s[h / x](x)=h$ and $s[h / x](y)=s(y)$ if $y \neq x$.

A constraint is a set of ground assignments and we let $C$, ranged over by $c$, be the set of all constraints. The operator $\exists x$ over a constraint $c$ makes a variable $x$ local in $c$, and is defined as $\exists x . c=\{s[h / x] \mid h \in \mathcal{H}, s \in c\}$. Of course, the ordering of $\exists x$ 's in a constraint is irrelevant. Thus, we can conveniently write $\exists X$ in place of $\exists x_{1}, \ldots, \exists x_{n}$, for $X=\left\{x_{1}, \ldots, x_{n}\right\}$. The support of a constraint $\operatorname{supp}(c)$ is the minimum set $\mathcal{W}$ of variables such that $x \in \mathcal{W}$ implies that $\exists x . c \neq c$. Intuitively, the support of a constraint $c$ contains all the variables which are relevant for $c$. The notion of support corresponds to the concept of set of free variables in process calculi. We abbreviate the notation for constraints by disregarding variables which are not in the support. Hence, by $(x=y)$ we write the constraint $c$ with support $\{x, y\}$ that contains all assignments to the same ground term for $x$ and $y$, namely $c=\left\{s_{1}, s_{2}, \ldots\right\}$ such that $s_{i}=\left[h_{i} / x, h_{i} / y, h_{i}^{\prime} / z, \ldots\right]$, where $z \in \mathcal{V} \backslash\{x, y\}$. Similarly, by $(x=$ end $)$ we denote the constraint consisting of all the assignments that map $x$ to end. The set $C$ can be proved to be a named constraint semiring [7] by taking product $\otimes$, sum $\oplus, 0$, and 1 as follows:

$-c \oplus d=c \cup d$

$-c \otimes d=c \cap d$

- The bottom element 0 is the empty set;

- The top element 1 is the constraint with empty support, i.e. the set of all ground assignments.

We also define a recursive operator $r e c_{x} \mathrm{c}$ over constraints. Recursive constraint variables $c_{y}$ contain (except for the binding construct) an extra variable that is used when unfolding the constraint. All occurrences of a $c_{y}$ in $c$ are bound by $r e c_{x} \mathrm{c}$ in $r e c_{x} \mathrm{c} . c$, independently of the variable appearing in the subscript. Recursive constraints are folded/unfolded using the following equation:

$$
r e c_{x} \text { c. } \exists X . c=\exists X . c\left[c_{i} / c_{y_{i}} \mid i \in I\right]
$$

with $c_{i}=r e c_{y_{i}}$ c. $\exists X . c\left[y_{i} / x\right]$ and $y_{i}$ for $i \in I$ the variables occurring as indexes of $\mathrm{c}$ in $c$. The (solution of the) recursive constraint $r e c_{x} c . c$ can be defined as the least fixpoint associated to the above recursive equation, which exists because the operations on constraints are continuous and are defined over a domain that is a lattice.

It can be proved that finitary constraints like those ones generated by the compilation in $\$ 4$ have a corresponding logic program [16]. As an example, consider a recursive constraint that amounts to assigning to a variable $z$ an arbitrary number of $\alpha$ 's followed by $\beta$ and, subsequently, by end. Namely:

$$
\begin{aligned}
c= & r e c_{z} \mathrm{c} \cdot \exists x_{1}, x_{2}, y_{1}, y_{2} \cdot\left(\left(z=x_{1}\right) \oplus\left(z=y_{1}\right)\right) \otimes \\
& \left(x_{1}=\beta\left(x_{2}\right)\right) \otimes\left(x_{2}=\text { end }\right) \otimes\left(y_{1}=\alpha\left(y_{2}\right)\right) \otimes c_{y_{2}}
\end{aligned}
$$


The logic program corresponding to $c$ is given by the clauses below plus the goal $\mathrm{F}(z)$ :

$$
\begin{aligned}
& \mathrm{F}\left(\mathrm{x}_{1}\right):-\mathrm{G}_{1}\left(\mathrm{x}_{1}\right) \\
& \mathrm{F}\left(\mathrm{y}_{1}\right):-\mathrm{H}_{1}\left(\mathrm{y}_{1}\right) \\
& \mathrm{G}_{1}\left(\beta\left(\mathrm{x}_{2}\right)\right):-\mathrm{G}_{2}\left(\mathrm{x}_{2}\right) \\
& \mathrm{G}_{2}(\text { end }) . \\
& \mathrm{H}_{1}\left(\alpha\left(\mathrm{y}_{2}\right)\right):-\mathrm{H}_{2}\left(\mathrm{y}_{2}\right) \\
& \mathrm{H}_{2}\left(\mathrm{y}_{2}\right):-\mathrm{F}\left(\mathrm{y}_{2}\right)
\end{aligned}
$$

Note that the constraint $c$ is generated by compiling the process of Example 4 in $\S 4$.

\subsection{Syntax and Semantics}

The syntax of target calculus (see Table 3) is analogous to the syntax of the source calculus apart for the introduction in the target processes of constraints $c \in \mathcal{C}$, as defined in $\S 3.1$, over possibly restricted variables. A service calls $\bar{\square}\langle x\rangle$ includes the 'root' variable $x$ of the constraint representing the interaction offered by the client to the invoked service. The process check $c_{1} . P_{1}$, check $c_{2} \cdot P_{2}$ generalises the external choice by evolving to the process $\mathrm{P}_{i}$ if $c_{i} \otimes c \neq 0$, with $c$ the current constraint and $i=1,2$.

We use $(X)$ where $X=\left\{x_{1}, \ldots, x_{n}\right\}$ to denote the restriction of the variables $x_{1}, \ldots, x_{n}$. We also write $\left(x_{1}, \ldots, x_{n}\right)$ for $\left(\left\{x_{1}, \ldots, x_{n}\right\}\right)$. Throughout the paper, we simplify constraints by implicitly assuming that restriction on constraints coincides with existential operator (namely, $(x) \mathrm{P} \mid c$ has the same meaning of $\mathrm{P} \mid \exists x . c$, if $x$ does not belong to the free variables of $P$ ), that restriction can be moved in and out of boxes, and that restrictions of variables not occurring in a term can be erased.

Akin to recursive constraints, recursive processes are also decorated with a source process recursion variable $p$. All occurrences of $\mathrm{p}_{x}^{p}$ in R are bound by $r e c_{y} \mathrm{p}^{p}$ in $r e c_{y} \mathrm{p}^{p}$. R. We identify recursive processes via the following fold/unfold equation

$$
\operatorname{rec}_{x} \mathrm{p}^{p} .(X) c|\mathrm{P}=(X) c| \mathrm{P}\left[\mathrm{P}_{i} / \mathrm{p}_{y_{i}}^{p} \mid i \in I\right]
$$

where $\mathrm{P}_{i}=r e c_{y_{i}} p .(X) c\left[y_{i} / x\right] \mid \mathrm{P}\left[y_{i} / x\right]$ and $y_{i}$ for $i \in I$ are all the variables occurring as indexes of $\mathrm{p}^{p}$ in $\mathrm{P}$.

Target client processes

$\mathrm{P}:=\bar{\square}\langle x\rangle . \mathrm{P}|\bar{\alpha} \cdot \mathrm{P}|\left(\right.$ check $c_{1} \cdot \mathrm{P}_{1}$, check $\left.c_{2} . \mathrm{P}_{2}\right)\left|\mathrm{p}_{x}^{p}\right| r e c_{x} \mathrm{p}^{p} . \mathrm{R}|\mathrm{R}| \bar{\nabla} . \mathrm{P}|\mathbf{0} \quad \mathrm{R}:=(X) c| \mathrm{P}$

Target service processes

$$
Q:=\alpha \cdot Q|Q+Q| \otimes
$$

Table 3. Syntax of target client and service processes.

A target client has the shape $(X) c \mid \bar{\square}\langle x\rangle$.P. We use $\mathrm{T}$ to range over target clients. A target service has the shape $(X) c \mid \square\langle x\rangle$.Q. We use $S$ to range over target services. Target systems $\vee$ are the same as source systems apart for the fact that the parallel composition of a client and a service includes a constraint and that a set $X$ of variables can be restricted in a box (where restricted variables are bound in their scope):

$$
\mathrm{U}::=c|\mathrm{P}| \mathrm{Q}|(X)[\mathrm{U}]| \mathrm{Q} \quad \vee::=\mathrm{T}|(X)[\mathrm{U}]|(X) c \mid \mathbf{0}
$$

Assume a set of available target services $\mathbb{S}_{t}$. We start by characterising the shape of the initial state of the LTS, which plays a key role in our theory as the existence of the initial state is meant to guarantee absence of deadlocks. To this purpose, we first define a function $I(-)$ that applied to a target client gives the set $Y$ of sets of variables 
which start the service calls. Each set of $Y$ contains the variables corresponding to a different execution path. Note that $Y$ cannot be infinite because recursive clients cannot have nested calls. For $Y$ a collection of sets, we let $\{x\} \uplus Y=\{\{x\} \cup Z \mid Z \in Y\}$.

$$
\begin{aligned}
& I(\bar{\square}\langle x\rangle . \mathrm{P})=\{x\} \uplus I(\mathrm{P}) \quad I(\bar{\alpha} . \mathrm{P})=I(\mathrm{P}) \quad I(\mathbf{0})=\{\emptyset\} \quad I(\bar{\nabla} \cdot \mathrm{P})=I(\mathrm{P}) \\
& I\left(\left(\text { check } c_{1} \cdot \mathrm{P}_{1} \text {, check } c_{2} \cdot \mathrm{P}_{2}\right)\right)=I\left(\mathrm{P}_{1}\right) \cup I\left(\mathrm{P}_{2}\right) \quad I\left(\mathrm{p}_{x}^{p}\right)=\{\emptyset\} \quad I\left(\operatorname{rec}_{x} \mathrm{p}^{p} \cdot \mathrm{R}\right)=\{\emptyset\}
\end{aligned}
$$

Assume a client $T$ and a set of available services $\mathbb{S}_{t}$. Suppose

$$
\begin{aligned}
\mathrm{T} & =(X) c_{0} \mid \bar{\square}\langle x\rangle . \mathrm{P} & \mathbb{S}_{t} & =\left\{\left(X_{i}\right) c_{i}\left|\square\left\langle x_{i}\right\rangle \cdot \mathrm{Q}_{i}\right| i \in I\right\} \\
I(\bar{\square}\langle x\rangle . \mathrm{P}) & =Y & d_{Y} & =\bigoplus_{Z \in Y} \otimes_{z \in Z} \bigoplus_{i \in I} \exists X_{i} \cdot c_{i} \otimes\left(z=x_{i}\right)
\end{aligned}
$$

If $c_{0} \otimes d_{Y} \neq 0$ then $\operatorname{start}\left(\mathrm{T}, \mathbb{S}_{t}\right)=\left(X \cup \bigcup_{Z \in Y} Z\right) c_{0} \otimes d_{Y} \mid \bar{\square}\langle x\rangle . \mathrm{P}$ is the initial state of the LTS in Table 4.

The consistency check $c_{0} \otimes d_{Y}$ amounts to say that for at least one set $Z \in Y$, every service call corresponding to a $z \in Z$ can successfully interact with a service in $\mathbb{S}_{t}$. Remark that rule $(t$-call $)$ is applied with $c$ being the product of $c_{0}$, the constraint of the client, and the constraints $d_{Y}$ of services interacting with service calls. Hence, the condition $c \otimes d \neq 0$ not only ensures that the interaction with the actual service will be successful, but also that any subsequent service call will find a suitable service.

In Table 4, $\varphi \in\left\{[, \bar{\nabla}\} \cup \overline{\mathcal{A}}, \psi \in\{[],\} \cup \mathcal{A}, \mathrm{Q} \downarrow_{\square}\right.$ is defined as for source processes, $\mathrm{W}$ stands for $c \mid \mathrm{P}$ or $(X)[\mathrm{U}]$, and the symmetric rule with respect to + has been omitted. The rules are the same as their homologous ones in the source calculus, taking into account that systems contain constraints. Rule ( $t$-check) activates a process continuation $\mathrm{P}_{i}$ only if the corresponding guard is consistent with the constraint store (condition $\left.c \otimes c_{i} \neq 0\right)$.

Notably, recursion of target clients is useful also if initial states choose finite sets of finite services, since the target calculus mimics all reductions of the source calculus (Theorem 4).

We define $\mathrm{V} \stackrel{\sigma}{\Longrightarrow}$ as expected. A system $\mathrm{V}$ is satisfied if it is of the shape $(X) c \mid \mathbf{0}$ for some $X, c$. We define $\mathrm{V} \Downarrow^{\text {must }}$ if either $\mathrm{V}$ is satisfied or for all $\sigma, \mathrm{V}^{\prime}$ such that $\mathrm{V} \stackrel{\sigma}{\Longrightarrow} \mathrm{V}^{\prime}$ we have $V^{\prime} \Downarrow^{\text {must }}$, i.e. all computations terminate with a satisfied system.

$$
\begin{aligned}
& \frac{(X) d \mid \square\langle x\rangle \cdot \mathrm{Q} \in \mathbb{S}_{t} \quad c \otimes d \neq 0}{c \mid \bar{\square}\langle x\rangle \cdot \mathrm{P} \stackrel{[}{\longrightarrow}(X)[c \otimes d|\mathrm{P}| \mathrm{Q}]}(t \text {-call }) \\
& c|\bar{\nabla} \cdot \mathrm{P} \stackrel{\square}{\longrightarrow} c| \mathrm{P}(\text { t-end }) \\
& c|\bar{\alpha} . P \stackrel{\bar{\alpha}}{\longrightarrow} c| \mathrm{P}\left(\text { t-action }_{C}\right) \\
& \alpha . Q \stackrel{\alpha}{\longrightarrow} Q\left(\text { t-action }_{S}\right)
\end{aligned}
$$

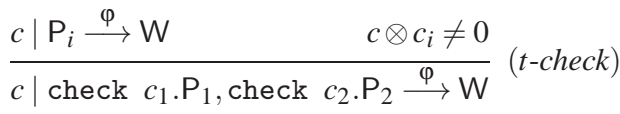

$$
\begin{aligned}
& \frac{\mathrm{Q} \stackrel{\alpha}{\longrightarrow} \mathrm{Q}^{\prime}}{\mathrm{Q}+\mathrm{Q}_{1} \stackrel{\alpha}{\longrightarrow} \mathrm{Q}^{\prime}} \text { (t-choice) } \\
& \frac{c|\mathrm{P} \stackrel{\bar{\alpha}}{\longrightarrow} c| \mathrm{P}^{\prime} \quad \mathrm{Q} \stackrel{\alpha}{\longrightarrow} \mathrm{Q}^{\prime}}{c|\mathrm{P}| \mathrm{Q} \stackrel{\alpha}{\longrightarrow} c\left|\mathrm{P}^{\prime}\right| \mathrm{Q}^{\prime}} \text { (t-interaction) } \\
& \frac{c|\mathrm{P} \stackrel{\bar{\otimes}}{\longrightarrow} c| \mathrm{P}^{\prime} \quad \mathrm{Q} \downarrow_{\otimes}}{[c|\mathrm{P}| \mathrm{Q}] \stackrel{]}{\longrightarrow} c \mid \mathrm{P}^{\prime}}(t-u p) \\
& \frac{\mathrm{U} \stackrel{\psi}{\longrightarrow} \mathrm{U}^{\prime}}{[\mathrm{U}] \stackrel{\psi}{\longrightarrow}\left[\mathrm{U}^{\prime}\right]}(t-b o x) \\
& \frac{\mathrm{V} \stackrel{\psi}{\longrightarrow} \mathrm{V}^{\prime}}{\mathrm{V}\left|\mathrm{Q} \stackrel{\psi}{\longrightarrow} \mathrm{V}^{\prime}\right| \mathrm{Q}}(\text { t-parallel }) \\
& \left.\frac{\mathrm{V} \stackrel{\psi}{\longrightarrow} \mathrm{V}^{\prime}}{(X) \mathrm{V} \stackrel{\psi}{\longrightarrow}(X) \mathrm{V}^{\prime}} \text { (t-restr }\right)
\end{aligned}
$$

Table 4. LTS for the target calculus 
Example 2. Take the client $\mathrm{T}=\left(x_{0}\right) c_{0} \mid \bar{\square}\left\langle x_{1}\right\rangle . \mathrm{P}$ with

$$
\begin{aligned}
& c_{0}=\left(x_{1}=\alpha\left(x_{0}\right)\right) \otimes\left(\left(x_{0}=\delta(\text { end })\right) \oplus\left(x_{0}=\rho(\text { end })\right)\right) \\
& \text { and } \quad \mathrm{P}=\bar{\alpha} \cdot\left(\operatorname{check}\left(x_{0}=\delta(\text { end })\right) \cdot \bar{\delta} \cdot \bar{\nabla} \cdot \mathbf{0}, \operatorname{check}\left(x_{0}=\rho(\text { end })\right) . \bar{\rho} \cdot \bar{\otimes} \cdot \mathbf{0}\right)
\end{aligned}
$$

and the service

$$
\mathrm{Q}=\left(y_{0}, y_{1}\right) d \mid \square\left\langle y_{1}\right\rangle . \alpha . \delta . \otimes \text { with } d=\left(y_{1}=\alpha\left(y_{0}\right)\right) \otimes\left(y_{0}=\delta(\text { end })\right)
$$

Then we get $I\left(\bar{\square}\left\langle x_{1}\right\rangle \cdot \mathrm{P}\right)=\left\{\left\{x_{1}\right\}\right\}=Y$ and $d_{Y}=\exists y_{0}, y_{1} . d \otimes\left(x_{1}=y_{1}\right)$.

The initial state of the client $T$ for the set of services $\{Q\}$ is

$$
\operatorname{start}(\mathrm{T},\{\mathrm{Q}\})=\left(x_{0}, x_{1}\right) c \mid \bar{\square}\left\langle x_{1}\right\rangle . \mathrm{P}
$$

where $c=c_{0} \otimes \exists y_{0}, y_{1} . d \otimes\left(x_{1}=y_{1}\right)$. It can reduce as follows:

$$
\begin{aligned}
& \left(x_{0}, x_{1}\right) c \mid \bar{\square}\left\langle x_{1}\right\rangle . \mathrm{P} \stackrel{[}{\longrightarrow}\left(x_{0}, x_{1}, y_{0}, y_{1}\right)[c \otimes d|\mathrm{P}| \alpha . \delta . \otimes] \\
& \stackrel{\alpha}{\longrightarrow}\left(x_{0}, x_{1}, y_{0}, y_{1}\right)[c \otimes d \mid \\
& \left.\operatorname{check}\left(x_{0}=\delta(\text { end })\right) \cdot \bar{\delta} . \bar{\nabla} . \mathbf{0}, \operatorname{check}\left(x_{0}=\rho(\text { end })\right) \cdot \bar{\rho} . \bar{\otimes} . \mathbf{0} \mid \delta . \otimes\right] \\
& \stackrel{\delta}{\longrightarrow}\left(x_{0}, x_{1}, y_{0}, y_{1}\right)[c \otimes d|\bar{\nabla} . \mathbf{0}| \otimes] \\
& \stackrel{]}{\longrightarrow}\left(x_{0}, x_{1}, y_{0}, y_{1}\right) c \otimes d \mid \mathbf{0}
\end{aligned}
$$

\section{Compilation}

We map the source calculus into the target calculus by adding constraints which take into account the interactions offered by the processes. This allows us to model the negotiation phase which precedes the choice of a service.

The compilation of services (Table 5) is simple, since the syntax of source and target processes is the same, when forgetting constraints and restrictions. This compilation adds an appropriate constraint for each process constructor by introducing a fresh variable $x$ which is equated to end for $\otimes$, to $\alpha(y)$ for $\alpha . Q$ (where $y$ is the variable introduced by the compilation of $Q$ ), to $x_{1}$ or $x_{2}$ for $Q_{1}+Q_{2}$ (where $x_{1}, x_{2}$ are the variables introduced by the compilation of $Q_{1}, Q_{2}$, respectively). Lastly the compilation of a service $\square . Q$ uses the variable $x$ introduced by the compilation of $Q$ to get $\square\langle x\rangle$.Q, where $\mathrm{Q}$ is the process obtained by compiling $Q$. All variables occurring in constraints are restricted in the resulting target service.

The compilation of clients (Table 6) is more complex, since client processes have nested calls, recursion and check expressions.

In order to deal with nested service calls we use a stack, represented as a list (nesting list), recording the variables which are the roots of the constraints in the encoding of the nested service calls which are still open and suspended (and then need to be closed); the head of this list contains the variable corresponding to the constraint root of the current call. We denote by nil the empty list, by $\ell$ an arbitrary list of variables or $\star$ and by

$$
\begin{aligned}
& \{[\otimes]\}_{x}=(x=\text { end }) \mid \otimes \\
& \{[Q]\}_{y}=(X) c \mid Q \text { implies }\{[\alpha \cdot Q]\}_{x}=(X \cup\{y\}) c \otimes e q(x, \alpha(y)) \mid \alpha \cdot Q \\
& \left\{\left[Q_{1}\right]\right\}_{x_{1}}=\left(X_{1}\right) c_{1} \mid Q_{1} \text { and }\left\{\left[Q_{2}\right]\right\}_{x_{2}}=\left(X_{2}\right) c_{2} \mid Q_{2} \text { imply }\left\{\left[Q_{1}+Q_{2}\right]\right\}_{x}= \\
& \quad\left(X_{1} \cup X_{2} \cup\left\{x_{1}\right\} \cup\left\{x_{2}\right\}\right) c_{1} \otimes c_{2} \otimes\left(\left(x=x_{1}\right) \oplus\left(x=x_{2}\right)\right) \mid Q_{1}+Q_{2} \\
& \{[Q]\}_{x}=(X) c \mid Q \text { implies }\{[\square \cdot Q]\}=(X \cup\{x\}) c \mid \square\langle x\rangle \cdot Q
\end{aligned}
$$

Table 5. Compilation of services 


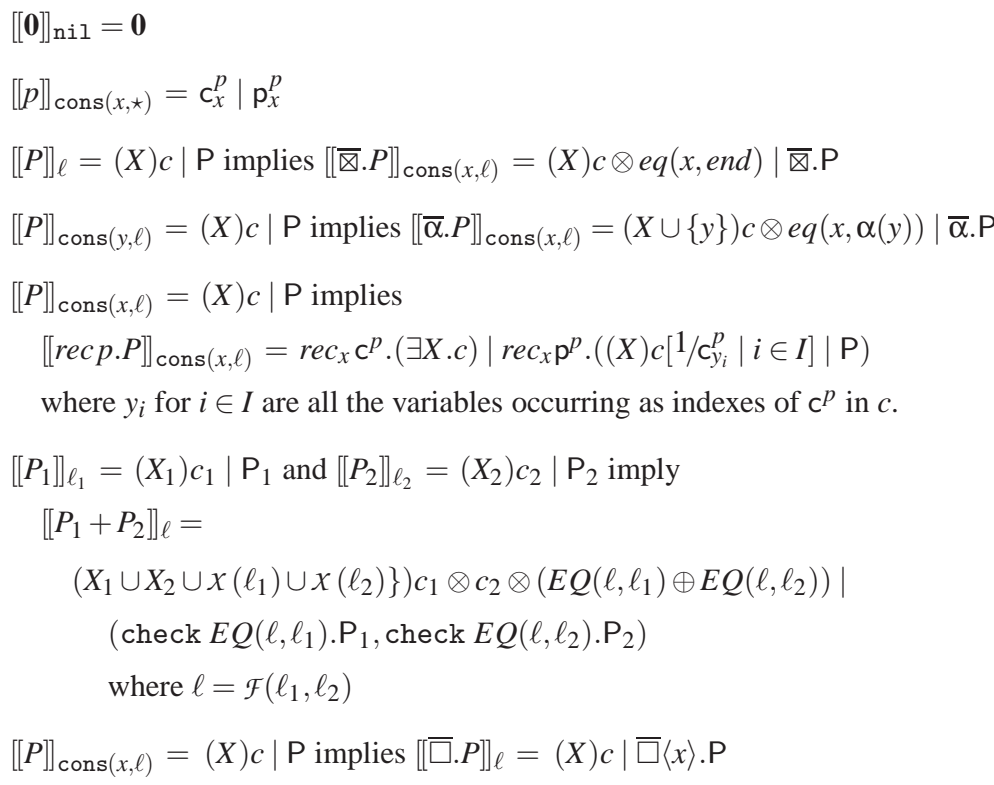

Table 6. Compilation of balanced clients

$\operatorname{cons}(x, \ell)$ the list with head $x$ and tail $\ell$. The nesting list of $\mathbf{0}$ is nil. For an interaction $\bar{\alpha} . P$ the nesting list is obtained just replacing the fresh variable $x$ to the variable $y$ which is the head of the nesting list in the compilation of process $P$. The compilation of the end $\bar{\nabla}$ of a service call corresponds (being the compilation bottom-up) to the resumption of the interaction with a service after the end of the nested interaction. Then we push a new fresh variable on the nesting list corresponding to the last action of the nested call.

When compiling a recursion variable the list of nested service is not yet known (being the compilation one-step) and so is replaced by the placeholder $\star$. Going on in the compilation process, however, we check (via the function $E Q$ defined in in Table 7) that the nesting lists of the suspended services in all branches of the recursive client are consistent (recall that no new service can be opened inside a recursive processes).

In the compilation of a choice we must assure that the suspended service calls will be resumed safely in the different branches (which could require different choices also in the paired services). This check is performed using the function $\mathcal{F}$ (see Table 7) which creates a new fresh nesting list starting from the nesting lists in the compilation of the two branches. The application of $\mathcal{F}$ to the lists $\ell_{1}, \ell_{2}$ is defined only if either $\ell_{1}, \ell_{2}$ have the same length or the shorter list terminates with $\star$. When defined the value of $\mathcal{F}\left(\ell_{1}, \ell_{2}\right)$ is a list of $n$ fresh variables, where $n$ is the maximum of the lengths of $\ell_{1}, \ell_{2}$, terminating with $\star$ if both $\ell_{1}, \ell_{2}$ terminate with $\star$ and with nil otherwise. Note that in a recursive process no $\bar{\nabla}$ can occur along a path ending in a recursion variable.

The compilation of a service call uses the head variable of the nesting list to compile the call and continues with the tail of the nesting list going then one level up in the call nesting. Note that the compilation of a balanced client has nil as final nesting list.

The process resulting from the compilation of a recursion variable is the parallel of a constraint variable and a process variable. This is so since in compiling recursive processes we need to generate both a recursive "global" constraint (taking into account 
all possible unfoldings of the process) and a recursive process including a "local" copy of its constraint (associated to each specific unfolding). The global recursive constraint characterises the process behaviour and is used in searching for a contract of one available service. The local copy of the constraints in each recursive call is used instead to keep track of the overall solution in the check branches. In the local copy of the constraint the occurrences of the recursion constraint variable (which are free) can be replaced by 1 since the consistency with the global solution is assured by the replacements of the process variables.

The compilation of a choice needs to check if one or both of the two branches agree with the interactions offered by services. This is accomplished by requiring that the list $\mathcal{F}\left(\ell_{1}, \ell_{2}\right)$ can be equated to $\ell_{1}$ or to $\ell_{2}$ considering that $\star$ can be equated to any list (see the definition of $E Q$ in Table 7).

All fresh variables which are introduced by the compilation are restricted in the resulting process, but for the variables occurring in the service calls. We use the function $x$ defined in Table 7 to restrict the variables of nesting lists in the compilation of choices.

It is easy to verify that all consistency checks in the premises of the rules of Table 4 are decidable when the target processes are obtained by compiling source processes. Indeed, recursive clients cannot have nested calls and the unfolding of the recursive constraints generated by the compilation of clients can be bounded by the maximum depth of the constraints generated by compiling the services, which are finite.

Example 3. The compilation of the source client $\bar{\square} \cdot \bar{\alpha} \cdot \bar{\square} \cdot \bar{\beta} \cdot(\bar{\gamma} \cdot \bar{\otimes} \cdot \bar{\delta} \cdot \bar{\otimes} \cdot \mathbf{0}+\bar{\mu} \cdot \bar{\otimes} \cdot \bar{\rho} \cdot \bar{\otimes} \cdot \mathbf{0})$, which is introduced in Example 1, is the following target client:

$$
\begin{aligned}
& \left(x_{0}, y_{0}, t_{1}, t_{0}, v_{1}, v_{0}, w_{1}, w_{0}, z_{1}, z_{0}\right)\left(x_{1}=\alpha\left(x_{0}\right)\right) \otimes\left(y_{1}=\beta\left(y_{0}\right)\right) \otimes \\
& \left(\left(x_{0}=t_{1}\right) \otimes\left(y_{0}=v_{1}\right) \oplus\left(x_{0}=w_{1}\right) \otimes\left(y_{0}=z_{1}\right)\right) \otimes\left(v_{1}=\gamma\left(v_{0}\right)\right) \otimes\left(v_{0}=\text { end }\right) \otimes \\
& \left(t_{1}=\delta\left(t_{0}\right)\right) \otimes\left(t_{0}=\text { end }\right) \otimes\left(z_{1}=\mu\left(z_{0}\right)\right) \otimes\left(z_{0}=\text { end }\right) \otimes\left(w_{1}=\rho\left(w_{0}\right)\right) \otimes\left(w_{0}=\text { end }\right) \mid \\
& \bar{\square}\left\langle x_{1}\right\rangle \cdot \bar{\alpha} \cdot \bar{\square}\left\langle y_{1}\right\rangle \cdot \bar{\beta} .\left(\operatorname{check}\left(x_{0}=t_{1}\right) \otimes\left(y_{0}=v_{1}\right) \cdot \bar{\gamma} \cdot \bar{\otimes} \cdot \bar{\delta} \cdot \bar{\nabla} \cdot \mathbf{0},\right. \\
& \left.\operatorname{check}\left(x_{0}=w_{1}\right) \otimes\left(y_{0}=z_{1}\right) \cdot \bar{\mu} \cdot \bar{\otimes} \cdot \bar{\rho} \cdot \bar{\otimes} \cdot \mathbf{0}\right)
\end{aligned}
$$

which can be simplified to

$$
\begin{aligned}
& \left(x_{0}, y_{0}\right)\left(x_{1}=\alpha\left(x_{0}\right)\right) \otimes\left(y_{1}=\beta\left(y_{0}\right)\right) \otimes \\
& \left(\left(x_{0}=\delta(\text { end })\right) \otimes\left(y_{0}=\gamma(\text { end })\right) \oplus\left(x_{0}=\rho(\text { end })\right) \otimes\left(y_{0}=\mu(\text { end })\right)\right) \mid \\
& \bar{\square}\left\langle x_{1}\right\rangle . \bar{\alpha} . \square\left\langle y_{1}\right\rangle . \bar{\beta} .\left(\operatorname{check}\left(x_{0}=\delta(\text { end })\right) \otimes\left(y_{0}=\gamma(\text { end })\right) \cdot \bar{\gamma} \cdot \bar{\nabla} \cdot \bar{\delta} . \bar{\otimes} . \mathbf{0},\right. \\
& \left.\quad \operatorname{check}\left(x_{0}=\rho(\text { end })\right) \otimes\left(y_{0}=\mu(\text { end })\right) \cdot \bar{\mu} . \bar{\nabla} . \bar{\rho} . \bar{\nabla} . \mathbf{0}\right)
\end{aligned}
$$

Note that this client can safely interact, for instance, with a set of (source) services including $\{\square . \alpha . \delta . \otimes, \square . \beta .(\gamma \cdot \otimes+v . \otimes)\}$, while he is stuck when the set of services is $\{\square . \alpha . \delta . \otimes, \square . \beta . \mu . \otimes\}$.

Example 4. Compiling the recursive process $\bar{\square}$.rec $p \cdot(\bar{\alpha} \cdot p+\bar{\beta} . \bar{\otimes} . \mathbf{0})$ we get, after some simplifications:

$$
\begin{aligned}
& r e c_{x} c^{p} \cdot \exists z \cdot((x=\alpha(z)) \oplus(x=\beta(\text { end }))) \otimes c_{z}^{p} \mid \\
& \square\langle x\rangle \cdot r e c_{x} p^{p} \cdot(z)((x=\alpha(z)) \oplus(x=\beta(\text { end }))) \mid \\
& \quad\left(\operatorname{check}(x=\alpha(z)) \cdot \bar{\alpha} \cdot p_{z}^{p}, \operatorname{check}(x=\beta(\text { end })) \cdot \bar{\beta} .0\right)
\end{aligned}
$$

The recursive constraint after two unfoldings and some simplifications becomes:

$$
\begin{gathered}
c=\exists z, z^{\prime} \cdot((x=\alpha(z)) \oplus(x=\beta(\text { end }))) \otimes\left(\left(z=\alpha\left(z^{\prime}\right)\right) \oplus(z=\beta(\text { end }))\right) \otimes \\
r e c_{z^{\prime}} c^{p} .\left(z^{\prime \prime}\right)\left(\left(\left(z^{\prime}=\alpha\left(z^{\prime \prime}\right)\right) \oplus\left(z^{\prime}=\beta(\text { end })\right)\right) \otimes c_{z^{\prime \prime}}^{p}\right)
\end{gathered}
$$

and the corresponding process: 


$$
\begin{gathered}
\bar{\square}\langle x\rangle \cdot(z)((x=\alpha(z)) \oplus(x=\beta(\text { end }))) \mid \\
\left(\operatorname{check}(x=\alpha(z)) \cdot \bar{\alpha} \cdot\left(z^{\prime}\right)\left(\left(z=\alpha\left(z^{\prime}\right)\right) \oplus(z=\beta(\text { end }))\right) \mid\right. \\
\left.\left(\operatorname{check}\left(z=\alpha\left(z^{\prime}\right)\right) \cdot \bar{\alpha} \cdot r e z_{z^{\prime \prime}} \mathrm{p}^{p} \ldots . ., \operatorname{check}(z=\beta(\text { end })) \cdot \bar{\beta} \cdot \mathbf{0}\right), \operatorname{check}(x=\beta(\text { end })) . \bar{\beta} . \mathbf{0}\right)
\end{gathered}
$$

If the service $(y) d \mid \square\langle y\rangle . \alpha . \beta . \otimes$, where

$$
d=\exists w_{0}, w_{1} .\left(y=\alpha\left(w_{0}\right)\right) \otimes\left(w_{0}=\beta\left(w_{1}\right)\right) \otimes\left(w_{1}=\text { end }\right)
$$

is available, then the constraint $\exists y . c \otimes d \otimes(x=y)$ is equal to $(x=\alpha(\beta($ end $)))$. This solution is determined only by the (proper) unfolding of the recursive constraint of the client and the constraint of the service. Once the solution is determined the constraint of each unfolding is used only to assure the correct choices in the check branches. These constraints are propagated via the unfolding of the recursive process and so no unfolding of the outermost recursive constraint is needed in the process reduction.

$$
\begin{gathered}
\mathcal{F}\left(\ell_{1}, \ell_{2}\right)= \begin{cases}\operatorname{cons}\left(x, \mathcal{F}\left(\ell_{1}^{\prime}, \ell_{2}^{\prime}\right)\right) & \text { if } \ell_{i}=\operatorname{cons}\left(x_{i}, \ell_{i}^{\prime}\right) \quad i=1,2 \\
\operatorname{cons}\left(x, \mathcal{F}\left(\ell_{1}^{\prime}, \star\right)\right) & \text { if } \ell_{1}=\operatorname{cons}\left(x_{1}, \ell_{1}^{\prime}\right) \text { and } \ell_{2}=\star \\
\operatorname{cons}\left(x, \mathcal{F}\left(\ell_{2}^{\prime}, \star\right)\right) & \text { if } \ell_{2}=\operatorname{cons}\left(x_{2}, \ell_{2}^{\prime}\right) \text { and } \ell_{1}=\star \\
\operatorname{iff} \ell_{1}=\ell_{2}=\operatorname{nil} \text { or } \ell_{1}=\text { nil and } \ell_{2}=\star \quad \text { where } x \text { is fresh } \\
\text { or } \ell_{2}=\operatorname{nil} \text { and } \ell_{1}=\star\end{cases} \\
\begin{array}{ll}
\text { if } \ell_{1}=\ell_{2}=\star \\
\text { otherwise. }
\end{array} \\
\text { undefined }
\end{gathered}
$$

Table 7. Auxiliary functions for the compilation of client choices

It is easy to verify that our compilation is successful for all source clients and processes, but for the case of unbalanced clients.

Theorem 1. Each balanced client and each service can be compiled.

More interesting (and more complex to prove) is the fact that given a compiled client $T$ and a set of available services $\mathbb{S}_{t}$, either $\operatorname{start}\left(\mathrm{T}, \mathbb{S}_{t}\right)$ is stuck or it always terminates.

Theorem 2. If $\mathrm{T}$ is obtained by compiling a source client and $\operatorname{start}\left(\mathrm{T}, \mathbb{S}_{t}\right) \stackrel{\sigma}{\Longrightarrow} \mathrm{V}$, then $\mathrm{V} \Downarrow^{\text {must }}$.

Comparing the reduction rules in Tables 2 and 4 it is easy to verify that the reductions of a target client obtained by compiling a source client correspond to reductions of the source client itself. We denote by $|\mathrm{V}|$ the source system obtained from the target system $\mathrm{V}$ by erasing all constraints and restrictions and by replacing checks by sums.

Theorem 3. (Soundness) If $\mathbb{S}_{t}$ is obtained by compiling the source services of $\mathbb{S}_{s}$ and $\operatorname{start}\left(\left[[T], \mathbb{S}_{t}\right) \stackrel{\sigma}{\Longrightarrow} \mathrm{V}\right.$, then $T \stackrel{\sigma}{\Longrightarrow}|\mathrm{V}|$. 
The aim of the compilation is to avoid deadlocks, but also to preserve all successful interactions. This is the content of the following theorem, whose proof requires a precise analysis of the relations between the reduct of a target system and the compilation of the reduct of a source system.

Theorem 4. (Completeness) If $\mathbb{S}_{t}$ is obtained by compiling the source services of $\mathbb{S}_{s}$ and $T \stackrel{\sigma}{\Longrightarrow} V$ and $V \Downarrow^{\text {may }}$, then there is $\mathrm{V}$ such that $\operatorname{start}\left(\left[[T], \mathbb{S}_{t}\right) \stackrel{\sigma}{\Longrightarrow} \vee\right.$ and $|\mathrm{V}|=V$.

\section{Optimised semantics}

In the previous Section we have shown that the semantics of the target calculus ensures stuck-freedom provided that for each service call of a client there is a service which is able to complete an interaction with the client successfully. Nevertheless, during a client-service negotiation, it is desirable to have a mean to select the services that offer more choices to the client (considering all choices equally satisfactory), among all services which can successfully complete an interaction. This property holds for the target LTS only if there is a single service. By contrast, in the general case in which there is more than a 'complying' service available, the target LTS gives no guarantee on this respect. For instance, let $T=\bar{\square} \cdot(\bar{\alpha} \cdot \bar{\nabla} \cdot \mathbf{0}+\bar{\beta} \cdot \bar{\otimes} . \mathbf{0})$ be a client and $S_{1}=\square \cdot(\alpha \cdot \otimes+\beta . \otimes)$ and $S_{2}=\square .(\alpha . \otimes+\gamma \cdot \otimes)$ be two services. According to the target semantics both $S_{1}$ and $S_{2}$ can be selected, as none of them would lead to a deadlock. Nevertheless, $S_{1}$ is somehow preferable as it additionally allows $T$ to exhibit action $\bar{\beta}$. Clearly for the client $\bar{\square} .(\bar{\alpha} . \bar{\otimes} . \mathbf{0}+\bar{\gamma} \cdot \bar{\nabla} . \mathbf{0})$ the service $S_{2}$ is better.

We define an optimised LTS that is obtained from the LTS given in Table 4 by replacing rule $(t$-call) by the following rule:

$$
\frac{(X) d \mid \square\langle x\rangle . \mathrm{Q} \in \mathbb{S}_{t} \quad \begin{array}{l}
c \otimes d \neq 0 \text { and } \nexists\left(X^{\prime}\right) d^{\prime} \mid \square\langle x\rangle . Q^{\prime} \in \mathbb{S}_{t} \text { s.t. } \\
c \otimes d \subset c \otimes d^{\prime}
\end{array}}{c \mid \square\langle x\rangle . \mathrm{P} \stackrel{[}{\longrightarrow}(X)[c \otimes d|\mathrm{P}| \mathrm{Q}]}(t \text {-call })
$$

Recall that constraints are sets of ground assignments, so they can be compared by inclusion. We denote by $\stackrel{\sigma}{\rightleftharpoons}$ reductions in the optimised LTS. Rule $\left(t\right.$-call $\left.l^{\prime}\right)$ ensures that a service $S$ is selected if it is one of the best possible services, namely such that there is no other service which offers the same successful interaction paths of $S$ and some more. Clearly in general we can get incomparable constraints, like for example for the client $T^{\prime}=\bar{\square} \cdot(\bar{\alpha} \cdot(\bar{\mu} \cdot \bar{\nabla} \cdot \mathbf{0}+\bar{\rho} \cdot \bar{\otimes} \cdot \mathbf{0})+\bar{\beta} \cdot \bar{\otimes} \cdot \mathbf{0}+\bar{\gamma} \cdot \bar{\otimes} \cdot \mathbf{0})$ and the services $S_{3}=$ $\square .(\alpha . \mu . \otimes+\beta . \otimes+\gamma . \otimes)$ and $S_{4}=\square . \alpha .(\mu . \otimes+\rho . \otimes)$. Note that the client $T^{\prime}$ has 3 successful paths choosing service $S_{3}$ and only 2 successful paths choosing service $S_{4}$, but these paths are incomparable. In such cases rule $\left(t\right.$-call $\left.l^{\prime}\right)$ chooses in a non deterministic way.

We prove that the new target semantics is optimal, in the sense that by choosing one of best services at each service call we obtain a set of services that guarantee the same interactions with more choices to a client. For simplicity we consider only unambiguous source processes, i.e. processes whose sums start with two different actions (modulo commutativity and associativity of sums). In order to formally state our result we note that each target system $\mathrm{V}$ which is obtained by reducing the compilation of a client and it is not satisfied has the shape $\mathrm{V}=\left(X_{n}\right)\left[\left(X_{n-1}\right) \ldots\left[\left(X_{0}\right)\left[c|\mathrm{P}| \mathrm{Q}_{0}\right] \mid \mathrm{Q}_{1}\right] \ldots \mathrm{Q}_{n}\right]$. This justifies the following definition. 
Definition 1. The constraint of the target system $\mathrm{V}$ (notation constr $(\mathrm{V})$ ) is inductively defined by:

$$
\begin{gathered}
\operatorname{constr}((X) c \mid \mathbf{0})=\mathrm{constr}(c|\mathrm{P}| \mathrm{Q})=c \\
\operatorname{constr}((X)[\mathrm{U}] \mid \mathrm{Q})=\mathrm{constr}((X)[\mathrm{U}])=\mathrm{constr}(\mathrm{U})
\end{gathered}
$$

Theorem 5. If $\mathrm{T}$ is obtained by compiling a source client and $\operatorname{start}\left(\mathrm{T}, \mathbb{S}_{t}\right) \stackrel{\sigma}{\longmapsto} \mathrm{V}$, then there is no $\mathrm{V}^{\prime}$ such that $\operatorname{start}\left(\mathrm{T}, \mathbb{S}_{t}\right) \stackrel{\sigma}{\Longrightarrow} \mathrm{V}^{\prime}$ and constr $(\mathrm{V}) \subset \operatorname{constr}\left(\mathrm{V}^{\prime}\right)$.

\section{Conclusion and Future Work}

In the paper we have augmented a client-service calculus with suitable constraints. A run time combination (multiplication in the simple cases) of client and service constraints guarantees that all and only the stuck-free interactions are possible. The constraints are exactly those of logic programming, and in fact a concrete representation of our constraints with logic programs and logic program combinations is straightforward. This property is comfortable from a theoretical point of view, since logic programs are well understood, but it is now appropriate to ask if it might be useful also from a practical point of view. We did not study the issue, which is outside the scope of this paper, but we can say that asking for satisfaction of the combined client-service constraint would be perfectly possible in any logic programming implementation, which would return an example of stuck-free interaction. Efficiency might depend on the exact way in which clauses are listed and parallel goals expanded. However it might be possible to devise an efficient algorithm (e.g. factorizing constraints and matching them top down breadth first) and to build a metainterpreter implementing the algorithm.

In the future, we plan to generalise the current target calculus by exploiting the formalism of Soft Constraint Logic Programming by Bistarelli, Montanari and Rossi [4]. In that paper, the ground semantics of a logic program (a goal and set of clauses) is not a set of ground assignments of the free variables of its goal, but rather a function from ground assignments to values of (another) constraint semiring. These values could give a measure of how acceptable the assignments are. Such functions, computed pointwise, form again a constraint semiring, and thus the formal treatment turns out simple and elegant. In particular the three semantics of logic programming (operational, denotational and model theoretical) can be defined also for soft constraint logic programming and proved equivalent. Specifically, in the context of the present paper a particular clientservice computation would not be only possible or impossible, but it could be assigned an acceptance weight, which might itself be structured by measuring the quality of service obtained in the interaction. For instance these weights could be taken into account in the reduction rules $(t$-call $)$ and $(t$-choice $)$ in order to allow only executions which maximise client's satisfaction.

In a different direction we will extend both source and target calculi with internal choices in order to model interactions in which one participant takes one branch independently from what is offered by the other participant.

\section{References}

1. L. Bettini, M. Coppo, L. D’Antoni, M. De Luca, M. Dezani-Ciancaglini, and N. Yoshida. Global progress in dynamically interleaved multiparty sessions. In CONCUR'08, volume 5201 of LNCS, pages 418-433. Springer, 2008. 
2. S. Bistarelli and F. Gadducci. Enhancing constraints manipulation in semiring-based formalisms. In ECAI'06, volume 141 of FAIA, pages 63-67. IOS Press, 2006.

3. S. Bistarelli, U. Montanari, and F. Rossi. Semiring-based constraint satisfaction and optimization. Journal of the ACM, 44(2):201-236, 1997.

4. S. Bistarelli, U. Montanari, and F. Rossi. Semiring-based constraint logic programming: syntax and semantics. ACM Transactions on Programming Languages and Systems, 23(1):129, 2001.

5. S. Bistarelli and F. Santini. A nonmonotonic soft concurrent constraint language for SLA negotiation. In CILC'08, volume 236 of ENTCS, pages 147-162. Elsevier, 2009.

6. M. Bravetti and G. Zavattaro. A theory of contracts for strong service compliance. Mathematical Structures in Computer Science, 19:601-638, 2009.

7. M. G. Buscemi and U. Montanari. Cc-pi: A constraint-based language for specifying service level agreements. In ESOP'07, volume 4421 of LNCS, pages 18-32. Springer, 2007.

8. M. G. Buscemi and U. Montanari. Qos negotiation in service composition. Journal of Logic and Algebraic Programming, 80(1):13-24, 2011.

9. G. Castagna, M. Dezani-Ciancaglini, and L. Padovani. On global types and multi-party sessions. In FMOODS\&FORTE'11, volume 6722 of LNCS, pages 1-28. Springer, 2011.

10. G. Castagna, N. Gesbert, and L. Padovani. A theory of contracts for web services. ACM Transactions on Programming Languages and Systems, 31, 2009. article n.19, pages 51.

11. M. Coppo and M. Dezani-Ciancaglini. Structured communications with concurrent constraints. In TGC'08, volume 5474 of $L N C S$, pages 104-125. Springer, 2009.

12. M. Dezani-Ciancaglini and U. de' Liguoro. Sessions and session types: an overview. In WSFM'09, volume 6194 of LNCS, pages 1-28. Springer, 2010.

13. M. Dezani-Ciancaglini, U. de' Liguoro, and N. Yoshida. On progress for structured communications. In TGC'07, volume 4912 of LNCS, pages 257-275. Springer, 2008.

14. N. Kobayashi. Type-Based Information Flow Analysis for the Pi-Calculus. Acta Informatica, 42(4-5):291-347, 2005.

15. C. Laneve and L. Padovani. The must preorder revisited: An algebraic theory for web services contracts. In CONCUR'07, volume 4703 of LNCS, pages 212-225. Springer, 2007.

16. J. W. Lloyd. Foundations of Logic Programming, 2nd Edition. Springer, 1987.

17. R. Milner. A Calculus of Communicating Systems. Springer, 1980.

18. R. Milner. Communicating and Mobile Systems: the Pi-Calculus. CUP, 1999.

19. L. Padovani. Contract-directed synthesis of simple orchestrators. In CONCUR'08, volume 5201 of LNCS, pages 131-146. Springer, 2008.

20. S. K. Rajamani and J. Rehof. Conformance checking for models of asynchronous message passing software. In $C A V^{\prime} 02$, volume 2402 of $L N C S$, pages 166-179. Springer, 2002.

21. V. A. Saraswat and M. C. Rinard. Concurrent constraint programming. In POPL'90, pages 232-245. ACM, 1990.

22. K. Takeuchi, K. Honda, and M. Kubo. An interaction-based language and its typing system. In PARLE'94, volume 817 of $L N C S$, pages 398-413. Springer, 1994.

23. V. T. Vasconcelos. Sessions, from types to programming languages. EATCS Bulletin, 103:53-73, 2011. 